See discussions, stats, and author profiles for this publication at: https://www.researchgate.net/publication/269192707

\title{
NOISE CORRECTION FOR SURFACE MEASUREMENTS
}

Chapter · July 2004

DOI: 10.1142/9789812702647_0029

2 authors, including:

Han Haitjema

KU Leuven

146 PUBLICATIONS 1,826 CITATIONS

SEE PROFILE

Some of the authors of this publication are also working on these related projects:

Project Solar Cell Manufacturing View project

CIRPedia View project 
3. D.Ichim, F.Pavese, J.Casas-Cubillos and C.Balle, Temperature, Its Measurements and Control in Science and Industry (D.Ripple Editor), AIP, New York, 2002, 429-

4. F.Pavese and P.Ciarlini: "Accurate modelling of translational bias and it pplication to reduction of thermophysical data series", Metrologia 27 (1990), 145-
Advanced Mathematical and Computational Tools in Metrology VI
Edited by P Ciarlini, M G Cox, F Pavese \& G B Rossi
O 2004 World Scientific Publishing Company (pp. 299-301)

NOISE CORRECTION FOR SURFACE MEASUREMENTS

H. HAITJEMA AND M.A.A. MOREL

Precision Engineering section,

Eindhoven University of Technology, PO Box 513,

5600 MB Eindhoven, The Netherlands

E-mail: : m.a.a.morel@itur..nl In roughness measurements often noise plays a role. Noise may give an offset i
measurement parameter as noise makes the parameter deviate away from zero. In this paper we propose a method to correct for noise bias for the surface parameter $S q$. By considering the decrease in $S q$ once an average over multiple measurements is made, an unbiased value for $S q$ is estimated by extrapolating the value to an infinite amount of measurements. It is shown that using this method for two measurements only, the truc is extended to obtain a complete 'noise-corrected' surface by considering the powe spectrum and the change of each Fourier component with averaging. Combining the two methods and considering the statistical significance of each Fourier component enables further reduction. Examples and simulations are shown for the calibration of roughnes
drive axis and surface measurements.

1. Method

1.1. Parameter extrapolation

The parameter $S q$ in surface metrology is a measure of power as it is the rms value of the surface. It is possible to use the mean square, $S q^{2}$ to extrapolate the $S q$ to a power level with reduced noise bias. As the noise is independent of the measurement, the power of a measurement consist of the power of the object and the power of noise [1]

$$
S q_{\text {measurement }}^{2}=S q_{\text {object }}^{2}+S q_{\text {noise }}^{2}
$$

If multiple measurements are taken, noise is reduced by the number of measurements $n$, but the power of the object remains:

$$
S q_{\text {mean }(n)}^{2}=S q_{\text {object }}^{2}+S q_{\text {noise }}^{2} / n
$$

Rearranging 2 and 3 gives the power of the object:

$$
S q_{o b j e c t}^{2}=\frac{n \cdot S q_{\text {mean }}^{2}-\left\langle S q_{\text {measurement }}^{2}\right\rangle}{n-1}
$$

In figure 1, the results are given of the extrapolation for a simulated block surface with an $S q$ of $1.381 \mu \mathrm{m}$. Measurement surface is $0.01 \mathrm{~mm}^{2}$, period 10 $\mu \mathrm{m}$ and amplitude $1 \mu \mathrm{m}$. A normal distributed noise with a standard deviation of 
$1 \mu \mathrm{m}$ is added. As the standard uncertainty in the extrapolated sets does not decrease, e.g. averaging two profiles gives $S q=1.547 \mu \mathrm{m} \pm 12 \mathrm{~nm}$ and the reduced pairs give $S q=1.376 \mu \mathrm{m} \pm 10 \mathrm{~nm}$, it is possible the calculated parameter is lower as the 'clean' surface, but within the uncertainty limits. The bias effect is observed in surface plates [2] and noted for rms values of wavefronts by Davies [3]. Davies uses a combinatorial method to remove the bias.

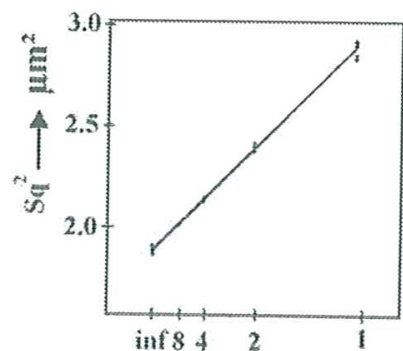

Number of measumements Figure 1. Extrapolated sets of two measurements compared to the mean of two, four and eight measurements and the singe results. The mean of eight files surface is in the upper right $(S q=1.419 \mu \mathrm{m})$, the randon phase exclusion surface is lower right $(S q$ $=1.374 \mu \mathrm{m})$.

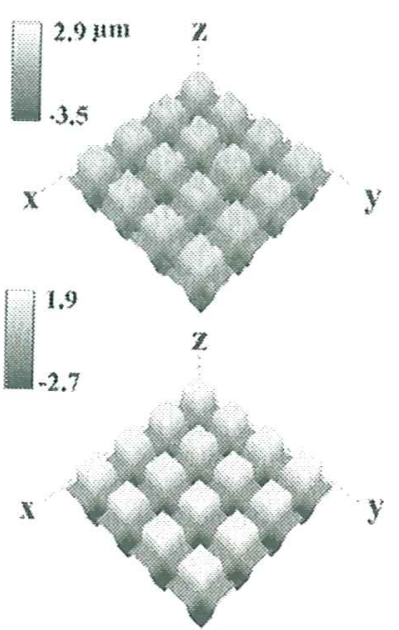

\subsection{Fourier reduction}

The next step is to obtain a complete reduced surface. First a Fourie transformation is performed. The power, the squared amplitude, of a single frequency also consists of an object and a noise part. Following formulas 1 to 3 , the amplitude spectrum of a noise reduced surface can be calculated. Inverse transformation is done with the phase of the mean of measurements. If a noisy frequency is encountered it is possible the power is reduced to a negative number. In that case the power of the frequency is set to zero.

\subsection{Random phase exclusion}

Even further reduction is possible. The Fourier terms are sorted by statistical importance. The more random a frequency, the larger the standard deviation in the mean of phases of that frequency will be and the less significant it is. Starting with the most significant frequency, $S q$ is calculated and compared to the extrapolated $S q_{\text {object }}$ calculated with equation 3. If the limit is not met the next frequency is added to the spectrum until $S q_{\text {surface }}$ meets $S q_{\text {object }}$.

\section{Calibration}

A drive axis of a roughness tester is calibrated with a randomizing method [4]. A reference plain is measured at 6 different positions. The reference and its phase is thus randomized while the axis remains constant. Using the noise correction and random phase exclusion methods, a plot of the axis is derived.

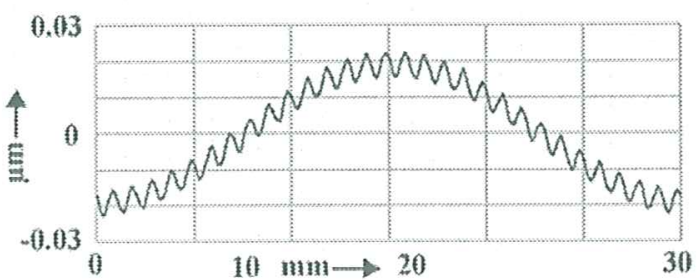

Figure 2. Calibration of drive axis of roughness tester with random phase exclusion.

\section{Acknowledgement}

This research is supported by Mitutoyo Nederland B.V.

\section{References}

1. C. Chatfield, Statistics for Technology, , Chapman \& Hall 1983, ISBN 0412-25340-2

2. H. Haitjema, Uncertainty propagation in Surface Plate measurements, in ISMQC 4th International symposium on dimensional metrology; Editors: H. Tikka, Tampere, Finland, 304-320, (1992)

3. A. Davies and. M.S. Levinson, Estimating the root mean square of a wavefront and its uncertainty, Applied Optics 40 (2001) pp 6203 - 6209.

4. C.J.Evans, R.J. Hocken and W.T. Estler, 'Self-calibration: reversal, redundancy, error separation and absolute testing', Annals of the CIRP, Vol 45/2, (1996) pp 617-634. 